PROCEEDINGS OF THE

AMERICAN MATHEMATICAL SOCIETY

Volume 127, Number 11, Pages 3153-3159

S 0002-9939(99)05155-2

Article electronically published on May 4, 1999

\title{
GROUP RINGS WHOSE SYMMETRIC ELEMENTS ARE LIE NILPOTENT
}

\author{
GREGORY T. LEE
}

(Communicated by Ronald M. Solomon)

\begin{abstract}
Let $F G$ be the group ring of a group $G$ over a field $F$, with characteristic different from 2 . Let $*$ denote the natural involution on $F G$ sending each group element to its inverse. Denote by $(F G)^{+}$the set of symmetric elements with respect to this involution. A paper of Giambruno and Sehgal showed that provided $G$ has no 2-elements, if $(F G)^{+}$is Lie nilpotent, then so is $F G$. In this paper, we determine when $(F G)^{+}$is Lie nilpotent, if $G$ does contain 2-elements.
\end{abstract}

\section{INTRODUCTION}

Throughout this paper, we will let $G$ be a group and $F$ a field with char $F=$ $p \neq 2$. The group ring $F G$ has a natural involution given by $\left(\sum_{g \in G} \alpha_{g} g\right)^{*}=$ $\sum_{g \in G} \alpha_{g} g^{-1}$. We will let $(F G)^{+}$denote the set of symmetric elements in $F G$. That is, $(F G)^{+}=\left\{\omega \in F G: \omega^{*}=\omega\right\}$. Similarly, $(F G)^{-}$will denote the set of skew elements; viz. $(F G)^{-}=\left\{\omega \in F G: \omega^{*}=-\omega\right\}$.

In any ring $R$, we define the Lie bracket on $R$ via $[x, y]=x y-y x$. Inductively, let $\left[x_{1}, \ldots, x_{n+1}\right]=\left[\left[x_{1}, \ldots, x_{n}\right], x_{n+1}\right]$. A subset $\Lambda$ of $R$ is said to be Lie nilpotent if, for some positive integer $n,\left[x_{1}, \ldots, x_{n}\right]=0$ for any $x_{1}, \ldots, x_{n} \in \Lambda$. The least such $n$ is called the index of nilpotence of $\Lambda$. In [4], Passi, Passman and Sehgal showed that $F G$ is Lie nilpotent if and only if $G$ is nilpotent and $p$-abelian, where char $F=p$. (Recall that $G$ is $p$-abelian if $G^{\prime}$ is a finite $p$-group, and 0-abelian will be taken to mean abelian.) In [1], Giambruno and Sehgal showed that if $G$ contains no 2-elements, then $(F G)^{+}$is Lie nilpotent if and only if $F G$ is Lie nilpotent.

We cannot expect this to remain true if $G$ has 2-elements. For instance, let $Q_{8}=\left\langle g, h \mid g^{4}=1, h^{2}=g^{2}, h^{-1} g h=g^{-1}\right\rangle$ be the group of quaternions. It is easy to verify that the symmetric elements of $F Q_{8}$ are commutative for any field $F$, since $\left(F Q_{8}\right)^{+}$is generated (as an $F$-module) by the elements $x+x^{-1}, x \in Q_{8}$ and the central elements 1 and $g^{2}$. However, if char $F \neq 2$, then $F Q_{8}$ itself is not Lie nilpotent, by [5, Theorem V.4.4]. Nevertheless, we will show that if $Q_{8} \nsubseteq G$, then $(F G)^{+}$is Lie nilpotent if and only if $F G$ is Lie nilpotent. Then, if $Q_{8} \subseteq G$, we will classify the groups $G$ for which $(F G)^{+}$is Lie nilpotent.

We thank the referee for the following observation, which ties our result in with representation theory. Suppose $G$ is finite and its order is not divisible by the

Received by the editors January 26, 1998.

1991 Mathematics Subject Classification. Primary 20C07, 16S34, 17B30.

The author is supported in part by a Province of Alberta Graduate Fellowship.

(C)1999 American Mathematical Society 
characteristic of $F$. It is easy to see that enlarging the field will not affect the Lie nilpotency of $(F G)^{+}$, so we may assume, without loss of generality, that $F$ is algebraically closed. Then, suppose $\tau: F G \rightarrow M_{n}(F)$ is an irreducible representation of $G$, where $M_{n}(F)$ is the ring of $n \times n$ matrices over $F$. We may define the contragredient representation $\mu: F G \rightarrow M_{n}(F)$ via $\mu(x)=\left(\tau(x)^{-1}\right)^{T}$ for all $x \in G$. We know that $\mu$ is an irreducible representation. Hence, if $\tau$ and $\mu$ are not equivalent, then $\tau \oplus \mu: F G \rightarrow M_{n}(F) \oplus M_{n}(F)$ is an epimorphism. It is simple to verify that for any $\alpha \in F G$, if $(\tau \oplus \mu)(\alpha)=(A, B)$, then $(\tau \oplus \mu)\left(\alpha^{*}\right)=\left(B^{T}, A^{T}\right)$. Thus, if $B=A^{T}$, then $(\tau \oplus \mu)(\alpha)=(\tau \oplus \mu)\left(\alpha^{*}\right)$, and therefore $(\tau \oplus \mu)\left(\frac{\alpha+\alpha^{*}}{2}\right)=(\tau \oplus \mu)(\alpha)$. Since $\tau \oplus \mu$ is surjective, it follows that $(F G)^{+}$maps homomorphically onto the set $\left\{\left(A, A^{T}\right): A \in M_{n}(F)\right\}$. Thus, $\tau\left((F G)^{+}\right)=M_{n}(F)$. Therefore, if $(F G)^{+}$is Lie nilpotent, then $M_{n}(F)$ is Lie nilpotent. By [5, Lemma V.2.2], $n=1$. Thus, if $\tau$ is any nonlinear irreducible representation of $F G$, then $\tau$ is equivalent to its contragredient. If, for instance, $F$ is the field of complex numbers, then the character of $\tau$ will have to be real. This eliminates many groups from consideration. However, we wish to allow $G$ to be infinite and $F G$ to be modular. We will use direct computations to solve the problem.

\section{Some LEMmata}

For any group $G$, we will let $\zeta$ (or $\zeta(G)$ ) denote its centre. Let us begin with

Lemma 1. If $(F G)^{+}$is Lie nilpotent, and char $F \neq 2$, then every element of order 2 in $G$ is central.

Proof. Let us assume that the characteristic of $F$ is $p>2$. Take $x, y \in G$ such that $o(x)=o(y)=2$. Then choosing $m$ such that $p^{m}$ is larger than the index of Lie nilpotence of $(F G)^{+}$, we have $0=[x, \underbrace{y, \ldots, y}_{p^{m} \text { times }}]=\left[x, y^{p^{m}}\right]$. Since $p$ is odd, $y^{p^{m}}=y$, and $x$ commutes with $y$.

Now, suppose $x, y \in G$, and $o(y)=2$. Then

$$
0=[x+x^{-1}, \underbrace{y, \ldots, y}_{p^{m} \text { times }}]=\left[x+x^{-1}, y^{p^{m}}\right]
$$

for some $m$. Once again, this gives us $\left[x+x^{-1}, y\right]=0$. Therefore, $x y=x^{-1} y, y x$, or $y x^{-1}$. If $x y=x^{-1} y$, then $x^{2}=1$, and the first case completes the proof. If $x y=y x$, there is nothing to do. If $x y=y x^{-1}$, then $(x y)^{2}=x y x y^{-1}=1$ and by the first case, $y$ and $x y$ commute. Therefore, $y x y=x y^{2}$, and $y x=x y$, as required.

If the characteristic of $F$ is zero, then $\mathbb{Z} G \subseteq F G$. Thus, $(\mathbb{Z} G)^{+}$is Lie nilpotent, and therefore so is $((\mathbb{Z} / 3 \mathbb{Z}) G)^{+}$. Using the result we have just proved, all elements of order 2 are central in $G$.

We will make use of the following observation several times.

Lemma 2. Let $F$ be a field with char $F \neq 2$, and let $G$ be a group such that $(F G)^{+}$ is Lie nilpotent. If, for some $a, b \in G$, we have $\left[a+a^{-1}, b+b^{-1}\right]=0$, then $a b$ is equal to one of the following: $b a, b^{-1} a, b a^{-1}$ or $b^{-1} a^{-1}$.

Proof. In the expression

$0=\left[a+a^{-1}, b+b^{-1}\right]=a b+a b^{-1}+a^{-1} b+a^{-1} b^{-1}-b a-b a^{-1}-b^{-1} a-b^{-1} a^{-1}$ 
the term $a b$ must be cancelled. For this to happen, it can either agree with one of the subtracted terms, or it can agree with at least two of the added terms (since the characteristic is not 2). In the latter case, we will either get $a b=a^{-1} b$ (in which case $a^{2}=1$ ) or $a b=a b^{-1}$ (in which case $b^{2}=1$ ), or possibly both. By Lemma 1 , this means that $a$ or $b$ is central, and $a b=b a$.

Lemma 3. Let $G=\langle a, b\rangle$, with $b^{-1} a b=a^{-1}$, and char $F \neq 2$. If $(F G)^{+}$is Lie nilpotent, then either $a^{2}=1$ (and $G$ is abelian) or $o(a)=4, o(b)=4 n$, where $n$ is an odd number, and $\left\langle a, b^{n}\right\rangle \simeq Q_{8}$.

Proof. If $a^{2}=1$, there is nothing to do, so let us assume $a^{2} \neq 1$. We begin by noting that, for any integer $i, a^{i} \in \zeta$ if and only if $a^{2 i}=1$. Also, $b^{2} \in \zeta$, but $b \notin \zeta$. Let the characteristic of $F$ be $p>2$. Suppose $o(b)=\infty$. Then $\left|\zeta^{2}\right|=\infty$, and the corollary in [1] tells us that $F G$ is Lie nilpotent. Thus by [5, Theorem V.4.4], $G$ is nilpotent, and $G^{\prime}$ is a finite $p$-group. Therefore, $\left\langle a^{2}\right\rangle$ contains a nontrivial central element. But $a^{-2}=b^{-1} a b a^{-1} \in G^{\prime}$, hence $o\left(a^{2}\right)=p^{m}$, for some $m \geq 0$. Thus, if $a^{2 i}$ is central for some $i$, then $a^{4 i}=1$, and since $a^{2}$ has odd order, $a^{2 i}=1$, which is a contradiction. Therefore, $b$ has finite order.

Now, since $b^{2} \in \zeta$, but $b \notin \zeta, b$ has order $2 k$ for some positive integer $k$. If $k$ is odd, then $b^{k}$ is central by Lemma 1 , and since $b^{2}$ is central, $b$ is central. This is impossible. Therefore, 4 divides $o(b)$. Let us write $o(b)=2^{r} n$, where $n$ is odd and $r \geq 2$. Then choosing $m$ such that $p^{m}$ exceeds the index of nilpotence of $(F G)^{+}$, we obtain

$$
0=[a b^{n}+b^{-n} a^{-1}, \underbrace{b^{n}+b^{-n}, \ldots, b^{n}+b^{-n}}_{p^{m} \text { times }}]=\left[a b^{n}+b^{-n} a^{-1}, b^{p^{m} n}+b^{-p^{m} n}\right] .
$$

By Lemma 2, we have four cases to consider.

(1) $a b^{n\left(1+p^{m}\right)}=b^{n p^{m}} a b^{n}$. Then $a b^{n p^{m}}=b^{n p^{m}} a$. Since $n p^{m}$ is odd, $b^{n p^{m}} \in \zeta$ implies $b \in \zeta$, and this is false.

(2) $a b^{n\left(1+p^{m}\right)}=b^{n\left(p^{m}-1\right)} a^{-1}$. Since $b^{n\left(p^{m}-1\right)}$ is central, we may deduce that $a b^{n\left(1+p^{m}\right)}=a^{-1} b^{n\left(p^{m}-1\right)}$. Hence $a^{2}=b^{-2 n}$.

(3) $a b^{n\left(1+p^{m}\right)}=b^{-n p^{m}} a b^{n}$. Because

$$
b^{-n p^{m}} a b^{n}=b^{-n p^{m}} a b^{n p^{m}} b^{n\left(1-p^{m}\right)}=a^{-1} b^{n\left(1-p^{m}\right)},
$$

we get $a b^{n\left(1+p^{m}\right)}=a^{-1} b^{n\left(1-p^{m}\right)}$. Hence $a^{2}=b^{-2 n p^{m}}$.

(4) $a b^{n\left(1+p^{m}\right)}=b^{-n\left(p^{m}+1\right)} a^{-1}$. Here, $a b^{n\left(1+p^{m}\right)}$ has order 2 and is central. Since $b^{n\left(1+p^{m}\right)}$ is central, $a$ is central, which is a contradiction.

Therefore, $a^{2}=b^{-2 n}$ or $b^{-2 n p^{m}}$. Either way, $a^{2} \in \zeta$, thus $o(a)=4$. Then $b^{4 n p^{m}}=1$ and since $o(b)=2^{r} n, o(b)=4 n$. We have $o(a)=o\left(b^{n}\right)=4, a^{2}=b^{-2 t n}$ for some odd number $t$ (so $a^{2}=b^{2 n}$ ), and $b^{-n} a b^{n}=a^{-1}$. Therefore, $\left\langle a, b^{n}\right\rangle \simeq Q_{8}$.

The characteristic zero case is handled just as in Lemma 1.

\section{The Case Without quaternions}

If $Q_{8} \nsubseteq \subseteq G$, then our proof is quite similar to that of [1].

Lemma 4. Let $G=\langle a, b\rangle$, and suppose $\left[a+a^{-1}, b+b^{-1}\right]=0$. If $Q_{8} \nsubseteq G$, and char $F \neq 2$, then $(F G)^{+}$is Lie nilpotent if and only if $G$ is abelian.

Proof. Suppose $[a, b] \neq 0$. By Lemma 2, $\left[a+a^{-1}, b+b^{-1}\right]=0$ yields $a b=b a$, $b a^{-1}, b^{-1} a$ or $b^{-1} a^{-1}$. The first case is contrary to our assumption. If $a b=b a^{-1}$ 
or $b^{-1} a$, then Lemma 3 gives us a contradiction. In the final case, $a b$ has order 2 and is therefore central. Hence $a^{2} b=a b a$, and therefore $a b=b a$. This is a contradiction.

Lemma 5. Suppose $Q_{8} \nsubseteq \subseteq G$ and $(F G)^{+}$is Lie nilpotent. Then

(1) if char $F=p>2$, then $G^{p^{m}} \subseteq \zeta$, for some $m$; and,

(2) if char $F=0$, then $G$ is abelian.

Proof. Let us prove (1). Choose $m$ such that $p^{m}$ is greater than the index of nilpotence of $(F G)^{+}$. Take $a, b \in G$. Then

$$
0=[a+a^{-1}, \underbrace{b+b^{-1}, \ldots, b+b^{-1}}_{p^{m} \text { times }}]=\left[a+a^{-1}, b^{p^{m}}+b^{-p^{m}}\right] .
$$

By Lemma 4, $a b^{p^{m}}=b^{p^{m}} a$. Therefore $b^{p^{m}} \in \zeta$.

To obtain $(2)$, we note that for all odd primes $q$, since $(\mathbb{Z} G)^{+}$is Lie nilpotent, $((\mathbb{Z} / q \mathbb{Z}) G)^{+}$is Lie nilpotent, hence $G^{q^{m}} \subseteq \zeta$ for some $m$. Since this holds for different primes $q, G$ is abelian.

Now, we are in a position to complete the case in which $G$ does not contain the quaternions. A little terminology is required. Let $F\left\{x_{1}, x_{1}^{*}, x_{2}, x_{2}^{*}, \ldots\right\}$ be the free algebra with involution. Then $0 \neq f\left(x_{1}, x_{1}^{*}, \ldots, x_{n}, x_{n}^{*}\right) \in F\left\{x_{1}, x_{1}^{*}, \ldots\right\}$ is called a *-polynomial identity for $F G$ if $f\left(a_{1}, a_{1}^{*}, \ldots, a_{n}, a_{n}^{*}\right)=0$ for all $a_{1}, \ldots, a_{n} \in F G$. Similarly, let $F\left\{x_{1}, x_{2}, \ldots\right\}$ be the free algebra over $F$. Then $0 \neq f\left(x_{1}, \ldots, x_{n}\right) \in$ $F\left\{x_{1}, x_{2}, \ldots\right\}$ is called a polynomial identity for $F G$ if $f\left(a_{1}, \ldots, a_{n}\right)=0$ for all $a_{1}, \ldots, a_{n} \in F G$. Also, let $\phi$ denote the $F C$-subgroup of a group $G$. Finally, we define $(a, b)=a^{-1} b^{-1} a b$ and inductively, $\left(a_{1}, \ldots, a_{n+1}\right)=\left(\left(a_{1}, \ldots, a_{n}\right), a_{n+1}\right)$. We have

Theorem 1. Suppose $Q_{8} \nsubseteq G$ and char $F=p \neq 2$. Then the following are equivalent:

(1) $(F G)^{+}$is Lie nilpotent;

(2) $F G$ is Lie nilpotent; and,

(3) $G$ is nilpotent and p-abelian.

Proof. Since (2) certainly implies (1), and the equivalence of (2) and (3) is given by [5, Theorem V.4.4], it will suffice if we can show that (1) implies (2).

If $F$ has characteristic zero, there is nothing left to do. Suppose char $F=p>2$. We know that $\left[x_{1}+x_{1}^{*}, \ldots, x_{r}+x_{r}^{*}\right]=0$ is a $*$-polynomial identity for $F G$, for some $r$. By [3, p. 196], FG satisfies a polynomial identity. By [5, Theorem V.1.1], $(G: \phi)<\infty$ and $\left|\phi^{\prime}\right|<\infty$. Since $G^{p^{t}} \subseteq \zeta$, for some $t$, by the last lemma, and $\zeta \subseteq \phi, G / \phi$ is a finite $p$-group.

To show that $G$ is nilpotent, it will suffice to show that $H=G / \zeta$ is nilpotent. Let $N=\phi / \zeta$. Then, since $G^{p^{t}} \subseteq \zeta$, we have $H^{p^{t}}=1$. Since $G / \phi$ is a finite $p$-group, so is $H / N$. Also, since $\phi^{\prime}$ is finite, so is $N^{\prime}$.

It is easy to see that $H / N^{\prime}$ acts as a finite $p$-group of automorphisms of $N / N^{\prime}$. Since $N^{p^{t}}=1$, [5, Lemma V.4.1] tells us that $\left(N / N^{\prime}, H / N^{\prime}, \ldots, H / N^{\prime}\right)=1$. That is, $(N, H, \ldots, H) \subseteq N^{\prime}$. Since $N^{\prime} / N^{\prime \prime}$ is finite and $H$ is a $p$-group, $H / N^{\prime \prime}$ acts as a finite $p$-group of automorphisms of $N^{\prime} / N^{\prime \prime}$. Again, by [5, Lemma V.4.1], $\left(N^{\prime}, H, \ldots, H\right) \subseteq N^{\prime \prime}$. Repeating this argument, we will eventually conclude that $(N, H, \ldots, H)=1$ (since $N^{\prime}$ is nilpotent, being a finite $p$-group). But $H / N$ is also 
nilpotent, so $(H, \ldots, H) \subseteq N$, and we conclude that $(H, \ldots, H)=1$. Thus, $H$ is nilpotent.

Now, since $G$ is nilpotent, and $G / \zeta$ is a $p$-group of bounded exponent by Lemma 5 , it follows from [5, Corollary I.4.3] that $G^{\prime}$ is a $p$-group. Suppose $G$ contains an element $x$ of infinite order. By Lemma 5 , some positive power of $x$ is central, and therefore $\left|\zeta^{2}\right|=\infty$. By the corollary in [1], we are done. Thus, we may assume that $G$ is a torsion nilpotent group. Then $G$ is the direct product $\prod_{\text {primes } q} P_{q}$, where each $P_{q}$ is the unique Sylow $q$-subgroup. Since $G^{\prime}$ is a $p$-group, $G^{\prime}=P_{p}^{\prime}$. Since $P_{p}$ has no 2-elements and $\left(F P_{p}\right)^{+}$is Lie nilpotent, the main result of [1] tells us that $P_{p}^{\prime}$ is finite. By [5, Theorem V.4.4], FG is Lie nilpotent.

\section{The CASE With QUATERnions}

We shall now determine what happens if $Q_{8} \subseteq G$.

Lemma 6. Assume char $F=p>2$, and suppose $\left(F\left(Q_{8} \times C\right)\right)^{+}$is Lie nilpotent, where $C$ is cyclic. Then $C$ has finite order $p^{m}$ or $2 p^{m}$ for some $m \geq 0$.

Proof. Let us write $Q_{8}=\langle g, h\rangle$, and $C=\langle c\rangle$. Then choosing $p^{m}$ to exceed the Lie nilpotency class, we obtain (in the usual manner)

$$
0=\left[g c+g^{-1} c^{-1}, h^{p^{m}} c^{p^{m}}+h^{-p^{m}} c^{-p^{m}}\right] .
$$

By Lemma 2, we have four cases to consider.

(1) $g h^{p^{m}} c^{p^{m}+1}=h^{p^{m}} g c^{p^{m}+1}$. Then $g h^{p^{m}}=h^{p^{m}} g$. But $p^{m}$ is odd, hence $h^{p^{m}}$ is not central in $Q_{8}$, which is a contradiction.

(2) $g h^{p^{m}} c^{p^{m}+1}=h^{p^{m}} g^{-1} c^{p^{m}-1}$. Then $c^{2}=1$.

(3) $g h^{p^{m}} c^{p^{m}+1}=h^{-p^{m}} g c^{1-p^{m}}$. Then $c^{2 p^{m}}=1$.

(4) $g h^{p^{m}} c^{p^{m}+1}=h^{-p^{m}} g^{-1} c^{-p^{m}-1}$. Then $\left(g h^{p^{m}}\right)^{2}=1$, but neither $g h$ nor $g h^{-1}$ is central in $Q_{8}$.

We are done.

We can now show that if an element does not have order $4 p^{m}$, then it must commute with the quaternions.

Lemma 7. Suppose $Q_{8}=\langle g, h\rangle \subseteq G$, char $F=p>2$, and $(F G)^{+}$is Lie nilpotent. If $b \in G$ and $b$ does not centralize $\langle g, h\rangle$, then $o(b)=4 p^{m}$ for some $m \geq 0$, and each of $\left\langle b^{p^{m}}, g\right\rangle$ and $\left\langle b^{p^{m}}, h\right\rangle$ is either abelian or isomorphic to $Q_{8}$.

Proof. Choosing $p^{k}$ larger than the Lie nilpotency index of $(F G)^{+}$, we obtain $0=$ $\left[b+b^{-1}, g^{p^{k}}+g^{-p^{k}}\right]=\left[b+b^{-1}, g+g^{-1}\right]$, since $g$ has order 4. Once again, Lemma 2 reduces the problem to four cases.

(1) $b g=g b$.

(2) $b g=g b^{-1}$. Then $g^{-1} b g=b^{-1}$. By Lemma 3, either $b^{2}=1$ and $b$ is central, or $o(b)=4$ and $\langle b, g\rangle \simeq Q_{8}$. This is the assertion with $m=0$.

(3) $b g=g^{-1} b$. Then $b^{-1} g b=g^{-1}$. Since $o(g)=4$, Lemma 3 tells us that $o(b)=4 n$ where $n$ is odd, and $\left\langle g, b^{n}\right\rangle \simeq Q_{8}$. Furthermore, $b^{4}$ clearly centralizes $\left\langle g, b^{n}\right\rangle$, and $o\left(b^{4}\right)=n$. Therefore, $\left\langle b^{4}\right\rangle \cap\left\langle g, b^{n}\right\rangle=\langle 1\rangle$. Thus, $\langle b, g\rangle=\left\langle g, b^{n}\right\rangle \times$ $\left\langle b^{4}\right\rangle \simeq Q_{8} \times C_{n}$. By Lemma 6 , since $n$ is odd, $n=p^{m}$ for some $m$.

(4) $b g=g^{-1} b^{-1}$. Then $(b g)^{2}=1$ and $b g$ is central. Hence $b^{2} g=b g b$, and $b g=g b$.

Thus, either $b g=g b$ or $o(b)=4 p^{m}$ for some $m \geq 0$ and $\left\langle b^{p^{m}}, g\right\rangle$ is either abelian or $Q_{8}$. The same can be said if we replace $g$ with $h$. 
The next two results narrow the possibilities down to $H \times P$, where $H$ is a Hamiltonian 2-group and $P$ is a $p$-group. Recall that a group is said to be Hamiltonian if it is non-abelian, but every subgroup is normal.

Lemma 8. Suppose $(F G)^{+}$is Lie nilpotent, where char $F=p>2$. If $Q_{8} \subseteq G$, then the 2-elements of $G$ form a normal subgroup which is a Hamiltonian 2-group.

Proof. Take $x, y \in G$ such that $o(x)=2^{r}$ and $o(y)=2^{t}$ for some $r, t \geq 0$. Choosing $m$ such that $p^{m}$ exceeds the nilpotency index of $(F G)^{+}$and $p^{m} \equiv 1\left(\bmod 2^{t}\right)$, we obtain $0=\left[x+x^{-1}, y^{p^{m}}+y^{-p^{m}}\right]=\left[x+x^{-1}, y+y^{-1}\right]$. Lemma 2 provides four cases.

(1) $x y=y x$.

(2) $x y=y x^{-1}$. Here, $y^{-1} x y=x^{-1}$ and by Lemma 3 , either $x$ and $y$ commute or $\langle x, y\rangle \simeq Q_{8}$

(3) $x y=y^{-1} x$. Then $x^{-1} y x=y^{-1}$, and either $x$ and $y$ commute or $\langle x, y\rangle \simeq Q_{8}$.

(4) $x y=y^{-1} x^{-1}$. In this case, $x y$ has order 2 and is therefore central. We get $y x y=x y^{2}$, hence $y x=x y$.

Thus, either $x$ and $y$ commute or the group they generate is isomorphic to $Q_{8}$. We conclude that $y^{-1} x y=x^{ \pm 1}$ and $\langle x, y\rangle$ is a 2-group. Therefore, the 2-elements form a subgroup $H$ whose every cyclic subgroup is normal in $H$. Since $H$ is not abelian, it is Hamiltonian. Clearly $H$ is normal in $G$.

Lemma 9. Suppose $F$ has characteristic $p>2,(F G)^{+}$is Lie nilpotent, and $Q_{8}=$ $\langle g, h\rangle \subseteq G$. Then $G \simeq Q_{8} \times E \times P$, where $E^{2}=1$ and $P$ is a p-group.

Proof. Suppose $x$ is an element of $G$ whose order is either infinity or an odd prime different from $p$. By Lemma 7, $x$ centralizes $\langle g, h\rangle$ and certainly $\langle x\rangle \cap\langle g, h\rangle=\langle 1\rangle$. Thus $Q_{8} \times\langle x\rangle \subseteq G$. By Lemma 6, we have a contradiction. By [2, Theorem 12.5.4], the Hamiltonian 2-groups are of the form $Q_{8} \times E$, where $E^{2}=1$. Thus, in view of Lemma 8 , it remains only to show that the $p$-elements form a subgroup. We know that every element has order $2^{r} p^{t}$ and since the Sylow 2-subgroup is $Q_{8} \times E, r=0$, 1 or 2 . Let $x$ and $y$ be $p$-elements of $G$, and suppose $\langle x, y\rangle$ contains a 2-element. By Lemma $7, x$ and $y$ commute with $\langle g, h\rangle$, but no element of order 4 in $Q_{8} \times E$ commutes with $\langle g, h\rangle$. Thus, the only 2-elements in $\langle x, y\rangle$ have order 1 or 2 . In particular, $Q_{8}$ does not lie in $\langle x, y\rangle$. But $(F\langle x, y\rangle)^{+}$is Lie nilpotent, and Theorem 1 tells us that $F\langle x, y\rangle$ is Lie nilpotent. Therefore, $\langle x, y\rangle$ is nilpotent and since $x$ and $y$ are $p$-elements, $\langle x, y\rangle$ is a $p$-group.

Finally, we have

Theorem 2. Suppose $Q_{8} \subseteq G$, and the characteristic of $F$ is not 2. Then $(F G)^{+}$ is Lie nilpotent if and only if either

(1) $\operatorname{char} F=p>2$ and $G \simeq Q_{8} \times E \times P$, where $E^{2}=1$ and $P$ is a finite p-group; or,

(2) $\operatorname{char} F=0$ and $G \simeq Q_{8} \times E$, where $E^{2}=1$.

Proof. Let us prove (1). Assume $(F G)^{+}$is Lie nilpotent. By Lemma 9, $G \simeq$ $Q_{8} \times E \times P$ where $E^{2}=1$ and $P$ is a $p$-group. Suppose $P$ is infinite. Then since $(F P)^{+}$is Lie nilpotent, Theorem 1 says that $P^{\prime}$ is finite. Thus $P / P^{\prime}$ is an infinite abelian $p$-group. Let $H=Q_{8} \times E \times\left(P / P^{\prime}\right)$. Then $(F H)^{+}$is Lie nilpotent, and $\zeta(H)^{2}$ is infinite. By the corollary in [1], $F H$ is Lie nilpotent. This is a contradiction to $\left[5\right.$, Theorem V.4.4], since $H^{\prime}$ is not a $p$-group. 
Conversely, suppose $G \simeq Q_{8} \times E \times P$, where $E^{2}=1$ and $|P|=p^{m}$, with $m \geq 0$. We claim that for all $\alpha_{1}, \ldots, \alpha_{p^{m}+1} \in(F G)^{+},\left[\alpha_{1}, \ldots, \alpha_{p^{m}+1}\right]=0$. Our proof is by induction on $m$. If $m=0$, then $G \simeq Q_{8} \times E$. In this case, $\left(F\left(Q_{8} \times E\right)\right)^{+}=$ $\left((F(E)) Q_{8}\right)^{+}$, and it is straightforward to verify that the symmetric elements commute. Suppose $m \geq 1$. Take $z \in \zeta(P)$ such that $o(z)=p$. By our inductive assumption, working in $F\left(Q_{8} \times E \times(P /\langle z\rangle)\right)$, we obtain $\left[\bar{\alpha}_{1}, \ldots, \bar{\alpha}_{p^{m-1}+1}\right]=0$. That is, $\left[\alpha_{1}, \ldots, \alpha_{p^{m-1}+1}\right] \in \Delta(G,\langle z\rangle)$, where $\Delta(G,\langle z\rangle)$ is the kernel of the natural homomorphism $F G \rightarrow F(G /\langle z\rangle)$. But $\Delta(G,\langle z\rangle)=\left(z^{2}-1\right) F G=\left(z-z^{-1}\right) F G$. Thus, $\left[\alpha_{1}, \ldots, \alpha_{p^{m-1}+1}\right]=\left(z-z^{-1}\right) \omega$, for some $\omega \in F G$. It is easy to see that $\left[(F G)^{+},(F G)^{+}\right] \subseteq(F G)^{-}$(the set of skew elements in $\left.F G\right)$, and $\left[(F G)^{-},(F G)^{+}\right] \subseteq$ $(F G)^{+}$. Thus, since $p^{m-1}+1$ is even, $\left(z-z^{-1}\right) \omega=\left[\alpha_{1}, \ldots, \alpha_{p^{m-1}+1}\right] \in(F G)^{-}$. Therefore, $\left(\left(z-z^{-1}\right) \omega\right)^{*}=-\left(z-z^{-1}\right) \omega$. Since $z-z^{-1}$ is both skew and central, we also have $\left(\left(z-z^{-1}\right) \omega\right)^{*}=-\left(z-z^{-1}\right) \omega^{*}$. Therefore, $\left(z-z^{-1}\right) \omega=\left(z-z^{-1}\right) \omega^{*}$, and it follows that $\left(z-z^{-1}\right) \omega=\left(z-z^{-1}\right) \beta_{1}$, where $\beta_{1}=\frac{\omega+\omega^{*}}{2}$ is symmetric. Then

$$
\begin{aligned}
{\left[\alpha_{1}, \ldots, \alpha_{2 p^{m-1}+1}\right] } & =\left[\left(z-z^{-1}\right) \beta_{1}, \alpha_{p^{m-1}+2}, \ldots, \alpha_{2 p^{m-1}+1}\right] \\
& =\left(z-z^{-1}\right)\left[\beta_{1}, \alpha_{p^{m-1}+2}, \ldots, \alpha_{2 p^{m-1}+1}\right] .
\end{aligned}
$$

As we have just observed, $\left[\beta_{1}, \alpha_{p^{m-1}+2}, \ldots, \alpha_{2 p^{m-1}+1}\right]=\left(z-z^{-1}\right) \beta_{2}$, for some $\beta_{2} \in(F G)^{+}$. (It is the Lie bracket of $p^{m-1}+1$ symmetric elements.) Iterating this argument, we obtain $\left[\alpha_{1}, \ldots, \alpha_{p^{m}+1}\right]=\left(z-z^{-1}\right)^{p} \beta_{p}$, for some $\beta_{p} \in(F G)^{+}$. But $\left(z-z^{-1}\right)^{p}=0$, and we are done.

To prove $(2)$, we note that if $(\mathbb{Z} G)^{+}$is Lie nilpotent, then so is $((\mathbb{Z} / q \mathbb{Z}) G)^{+}$for any odd prime $q$. By the first case, $G \simeq Q_{8} \times E \times P$, where $P$ is a finite $q$-group, for each odd prime $q$. Therefore $G \simeq Q_{8} \times E$. As we have observed before, the converse is trivial.

We conclude by mentioning that the paper of Giambruno and Sehgal also showed that if $G$ has no 2-elements and $(F G)^{-}$is Lie nilpotent, then so is $F G$. Our Theorem 1, however, will not hold for $(F G)^{-}$. As a counterexample, consider $D_{8}=\left\langle\sigma, \tau \mid \sigma^{4}=\tau^{2}=(\sigma \tau)^{2}=1\right\rangle$. It is clear that if char $F \neq 2$, then $\left(F D_{8}\right)^{-}$is commutative, but by [5, Theorem V.4.4], $F D_{8}$ is not Lie nilpotent.

\section{ACKNOWLEDGEMENT}

The author would like to express his thanks to his supervisor, Dr. Sudarshan Sehgal, both for proposing this problem and for all of his guidance along the way.

\section{REFERENCES}

1. A. Giambruno, S. K. Sehgal, Lie nilpotence of group rings, Comm. Algebra 21 (1993), 42534261. MR 94g:20008

2. M. Hall, The theory of groups, Macmillan, New York, 1959. MR 21:1996

3. I. Herstein, Rings with involution, Univ. of Chicago Press, Chicago, 1976. MR 56:406

4. I. B. S. Passi, D. S. Passman, S. K. Sehgal, Lie solvable group rings, Canad. J. Math. 25 (1973), 748-757. MR 48:4092

5. S. K. Sehgal, Topics in group rings, Marcel Dekker, New York, 1978. MR 80j:16001

Department of Mathematical Sciences, University of Alberta, Edmonton, Alberta, Canada T6G 2G1

E-mail address: glee@vega.math.ualberta.ca 